\title{
Influence on Power Quality Measurement of the Simultaneous Presence of Different Disturbances
}

\author{
Mañana, M; Eguíluz, L.I.; Lavandero, J.C.; Díez, G. \\ Department of Electrical Engineering \\ E.T.S.I.I., University of Cantabria \\ Avda. Los Castros s/n, 39006 Santander (Spain) \\ phone:+34 942201378, fax:+34 942201385, e-mail: mananam@unican.es
}

\begin{abstract}
Power Quality measurements involve not only problems related with the real use of recorders but also theoretical questions connected with functional and design aspects of the instrumentation. In addition, there are a rising number of standards covering topics from design to data analysis, so final users usually assume that they do not have to pay special attention to $\mathrm{PQ}$ instrumentation functionality when the device is designed according to approved standards. However, it is not unusual to find different disturbances at the same point and in the same time and standards do not say anything about their mutual influence on PQ index.

The main aim of this research work is to show the influence that having different disturbances (flicker and sags) simultaneously has on the final results provided by the instrumentation for each individual disturbance. The paper proposes a test system and a methodology. The results of various tests using commercial and research instrumentation are presented and some possible solutions to these problems are considered.
\end{abstract}

\section{Key words}

Power Quality, Flickermeter, Flicker, Harmonics, Sag, Disturbances.

\section{Introduction}

The European Standard EN 50160 [1] defines the power quality within which any customer can expect the voltage quality characteristics to remain at the customer's supply terminals in public electricity distribution networks under normal operating conditions. Similarly, the set of standards EN 61000-3 defines the quality conditions of the current demanded by non-linear loads. Both voltage and current should be measured by Power Quality instrumentation. There is also a set of standards entitled EN 61000-4 which defines the functional behaviour of this kind of recorder devices. The architecture of the instrumentation is well defined in the case of harmonics and interharmonics. The standard EN 61000-4-7 [2] is a general guide on harmonics and interharmonics measurements and instrumentation for power supply systems and equipment connected to them. This document focuses only on the measurement of harmonics and interharmonics from dc to $2500 \mathrm{~Hz}$ and the influence of any other kind of disturbances on the device is not considered.

Specifications in EN 61000-3-3 [3] and in other related IEC standards defining measuring instrumentation apply exclusively to $50-\mathrm{Hz}$ systems, but some technical groups are working in order to provide additional specifications suitable for use in $60-\mathrm{Hz}$ systems. Flicker is defined in terms of incandescent lamp behaviour because of common usage, at least in the past, and what is more important, because incandescent lamps exhibit greater sensitivity to voltage changes. Flicker may also be observed with fluorescent lamps and with other types of devices. This work is based on previous work performed by Koponen [4]

\section{Power Quality instrumentation under test}

The set of Power Quality Instrumentation devices under test (PQDUT) includes five flickermeters from four different companies. Four recorders are designed according to the standard EN 61000-4-15 [5] using a digital implementation. The last one is based on IEC 868 [6] and is implemented using analog filters.

Table I summarises some functional and design characteristics of the PQDUT.

\section{Flickermeter model}

The standard EN 61000-4-15 [5] defines the functional and design specifications of a flickermeter. The basic diagram of the instrumentation defined by the document is shown in Figure 1. This section provides a brief description of each block. More details are given in McKim [7].

Block 1-Input Voltage Adapter. The primary function of this block is to provide a normalised rms voltage to the 
next block, Block 2. The adapter includes an automatic gain control (AGC) circuit with a 10 to $90 \%$ stepresponse characteristic of 1 minute. Higher frequency voltage fluctuations pass through the AGC unattenuated, but long-term trends are effectively removed.

TABLE I. - PQDUT functional and design characteristics.

\begin{tabular}{|l|l|}
\hline Flickermeter & Description \\
\hline M_1 & $\begin{array}{l}\text { Three phases. } \\
\text { Digital. } \\
\text { EN 50160. }\end{array}$ \\
\hline M_2 & $\begin{array}{l}\text { Three phases. } \\
\text { Digital. } \\
\text { EN 61000-4-15. }\end{array}$ \\
\hline M_3 & $\begin{array}{l}\text { One phase. } \\
\text { Digital. } \\
\text { EN 61000-4-15. }\end{array}$ \\
\hline M_4 & $\begin{array}{l}\text { Three phases. } \\
\text { Digital. }\end{array}$ \\
\hline M_5 & Three phases. \\
& Analog. \\
IEC 868.
\end{tabular}

The functionality of Block 1 also includes a calibration generator. It is not clear whether the internal generator provides self calibration ability for analog instrumentation or simply a way to test its accuracy.

In addition, digital meters have enough calibration stability to eliminate the need for continuous verification.

The document does not consider the input transformer as part of Block 1. However, its function is closely linked because the transformer is also used to adapt the instrument input circuit to the nominal level of the measured voltage signal. Modern instrumentation may be designed without input transformers because variablegain differential amplifiers are widely used.

The output of Block 1 is also applied to an optional rms voltage-measuring circuit in order to register the voltage change characteristic time series that allows some parameters defined in the standard EN 61000-3-3 [3] to be defined.

Block 2-Demodulator. This block includes a squaring multiplier used as a demodulator. The main aim of this element is to recover modulating signals while simultaneously suppressing the main frequency carrier signal.

The output of Block 2 is applied to the input of Block 3. The only desired output from Block 2 are the components of frequency between 0.5 and $30 \mathrm{~Hz}$. Unwanted frequencies are eliminated by filters in Block 3 .

Block 3-Weighting Filters. Block 3 is designed to filter out of range frequencies. The block includes three filters connected in series and a ranging circuit. The first one is a first-order high-pass having the cutoff frequency set to $0.05 \mathrm{~Hz}$. The second one is a low pass filter with a cutoff frequency of $35 \mathrm{~Hz}$. Neither low nor high pass filters provide any kind of weighting. They have the aim of filtering the components between 0.05 and $35 \mathrm{~Hz}$. The third filter, meanwhile, provides a band-pass response centered at $8.8 \mathrm{~Hz}$. This filter weights the spectral components of the input signal within the frequency band of interest between $0.05 \mathrm{~Hz}$ and $35 \mathrm{~Hz}$. It models a part of the eye-brain set for an average human observer. This response peaks at $8.8 \mathrm{~Hz}$. The filter is very precisely specified by means of an equation documenting the required transfer function in the s-domain.

The ranging function included at the output of Block 3 is required for instruments using certain types of statistical classifiers in Block 5. This operation can be eliminated if nonlinear classifiers are used. In either case, the instrument must provide values of instantaneous flicker sensation within a range of 0.01 to 6400 in units of perceptibility threshold. The document defines full scale ranges corresponding to $\Delta \mathrm{U} / \mathrm{U}(\%)$ levels of $0.5,1,2,5$, 10 , and 20 . The values are defined with a requirement for a minimum resolution of 1 part in 64 within each range.

It is important to highlight that the relationship between flicker perceptibility and $\Delta \mathrm{U} / \mathrm{U}(\%)$ varies with frequency so there is no direct relationship between the two sets of ranges. Instead, the $\Delta \mathrm{U} / \mathrm{U}(\%)$ ranges are specified in order to ensure that the desired dynamic range for flicker sensation is maintained at any modulating frequency.

Block 4-Squaring Multiplier and First-Order Sliding Mean Filter. This block models the remainder of the eyebrain set for flicker perception. The squaring operator simulates nonlinear eye-brain response characteristics while the $300 \mathrm{~ms}$ first-order filter simulates perceptual storage effects in the brain. It can be implemented as a first-order low pass with a corner frequency of $0.53 \mathrm{~Hz}$.

The set of blocks 1 to 4 must produce an instantaneous flicker value of 1 for a list of sinusoidal and square modulations defined in the standard [5].

Optional computations can be performed with the output of Block 4 in order to smooth it or to use it for other purposes.

As a conclusion, blocks 2 to 4 model the filament-eyebrain set.

Block 5-Statistical Classifier. This block performs a statistical evaluation of the instantaneous flicker perception. It can also be considered as a model of human irritability in the presence of flicker stimulation. From a physiological point of view, flicker can be considered more tolerable if it occurs infrequently over short intervals. Fatigue and tolerance decrease in the presence of increasing level intensity and event duration.

The input to Block 5 includes an analog to digital converter (ADC) followed by a statistical classifier. Most modern instruments are digital so the ADC is placed at the beginning of Block 1.

The classifier itself is dedicated to obtaining the statistical information required to calculate short-term 


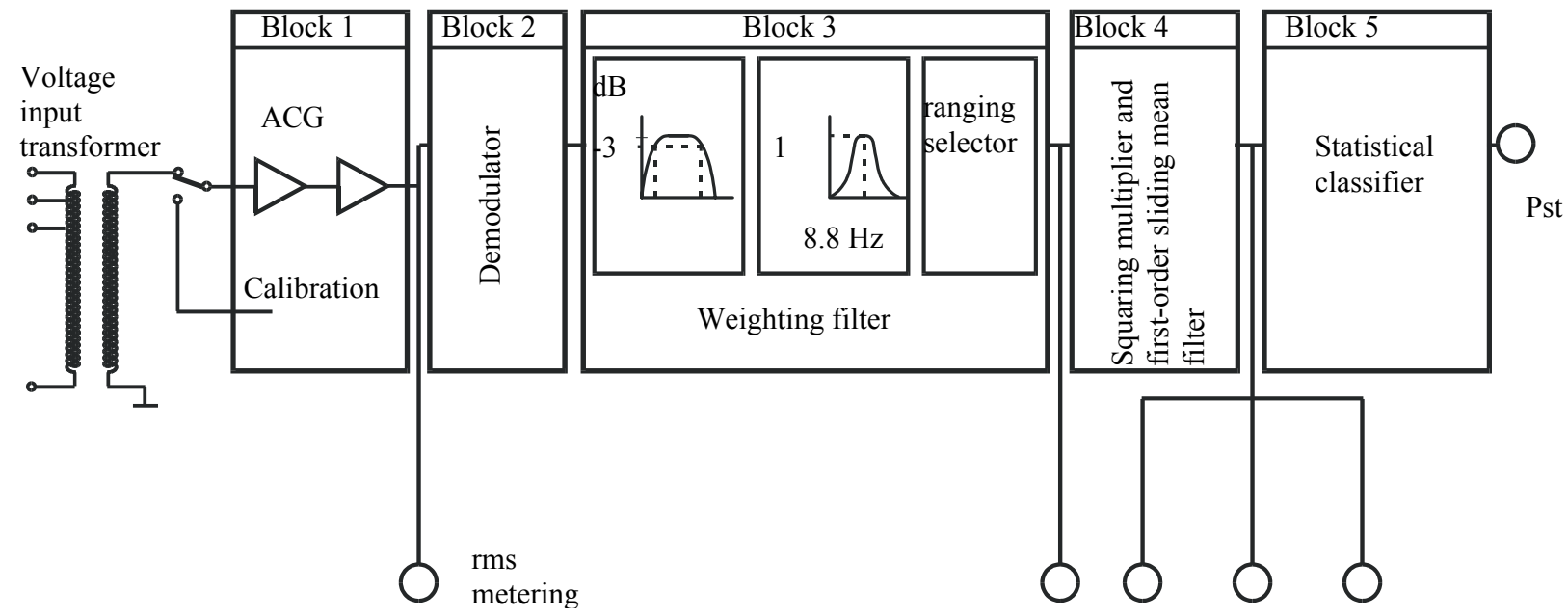

Auxiliar values for other purposes

Fig. 1. Flickermeter functional diagram according EN 61000-4-15 [4].

flicker severity (Pst), as well as long-term flicker severity (Plt).

Short-term flicker severity is computed using percentile values obtained from the statistical classifier. The statistical classifier implements a simple equation for calculating percentile values for instantaneous flicker sensation on a "time at level" basis. This is done for integration periods of different duration $[1,5,10$, or 15 minutes]. The 10 minutes period has been specified as the integration period for reference-grade compliance measurements. If we consider that instantaneous flicker perception is sampled at a rate of $200 \mathrm{~Hz}$ and that a 10minute integration period is used, 120000 individual samples of instantaneous flicker perception will be captured for each calculation of Pst.

Each sample is accumulated in the statistical classifier according to its level. The standard considers a minimum of 64 levels for the cumulative probability function. A set of percentiles may then be calculated using standard statistical methods and interpolation techniques. The levels can follow a linear or logarithmic distribution. Modern implementations use logarithmic distributions, usually with 1024 steps or more. This approach, together with an adequate ADC resolution, eliminates the need for the ranging function. Some research work has shown [7] that logarithmic classification avoids some accuracy problems that arise when applying linear distribution.

Finally it should be pointed out that percentile subscripts do not correspond to percentages of IFL values at lower values. They represent percentages of samples for which levels are exceeded. Long term flicker severity (Plt) is calculated from a set of 12 Pst values registered during 2 hours using 10 minute Pst integration period.

The overall model has been implemented using MatlabSimulink [8] in order to evaluate the influence of sags on each block. The model has been validated using the reference values proposed by the standard [5].
The behaviour of the theoretical model has been simulated using sags of different duration and amplitude. Figure 2 shows the instantaneous value of $\mathrm{I}_{\mathrm{FL}}$ for three sags: $(0 \%, 0.01 \mathrm{~s}),(50 \%, 0.01 \mathrm{~s})$ and $(80 \%, 0.01 \mathrm{~s})$.

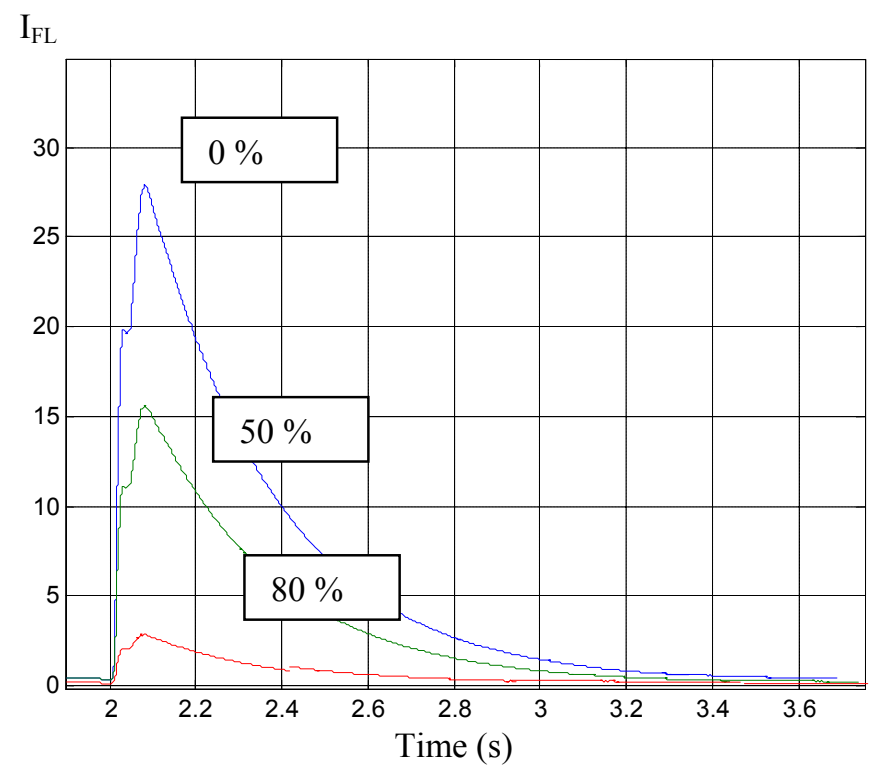

Fig. 2. IFL transient for a $0.01 \mathrm{~s}$ sags.

Figures 3 and 4 also show the instantaneous values of IFL for 0.05 and $0.1 \mathrm{~s}$ sags.

\section{Test system and methodology}

The Test System uses an arbitrary waveform power supply and test HP 6834B [9] that can be programmed in order to generate a $50 / 60 \mathrm{~Hz}$ sinusoidal voltage distorted with flicker.

The ac source combines three instruments in one unit as shown in Figure 5. The function generator produces waveforms with user defined amplitude, frequency, and 
shape. The power amplifier amplifies the function generator signal to produce the ac voltage. No power generation capabilities are needed because the flickermeters do not have any power consumption. The measurement functions range from a simple readback of rms voltage and current, to sophisticated capabilities such as waveform analysis.

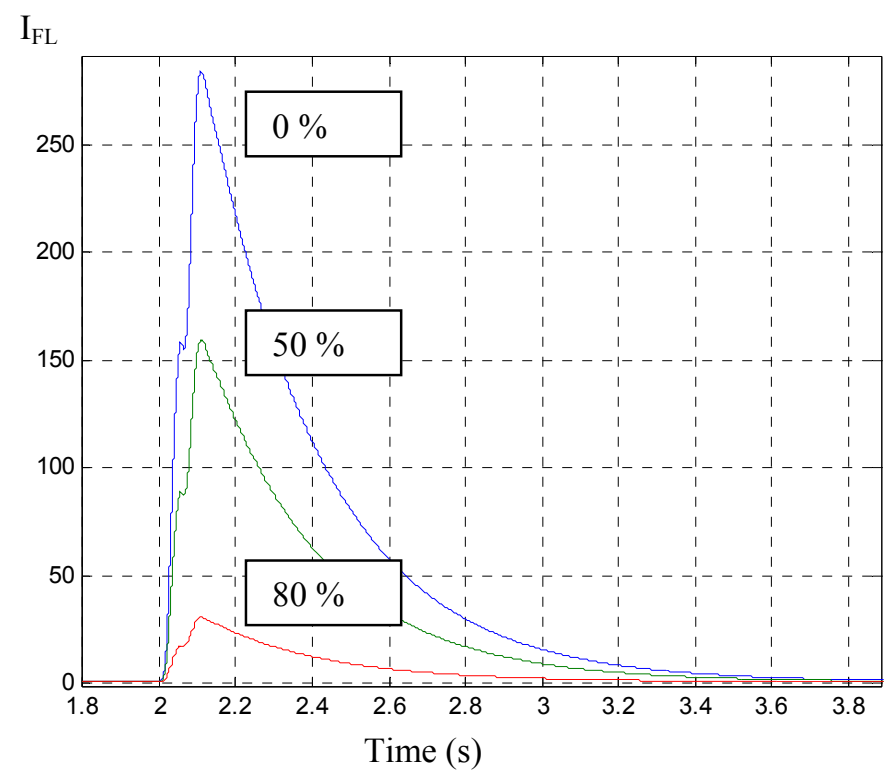

Fig. 3. IFL transient for a 0.05 s sags.

$\mathrm{I}_{\mathrm{FL}}$

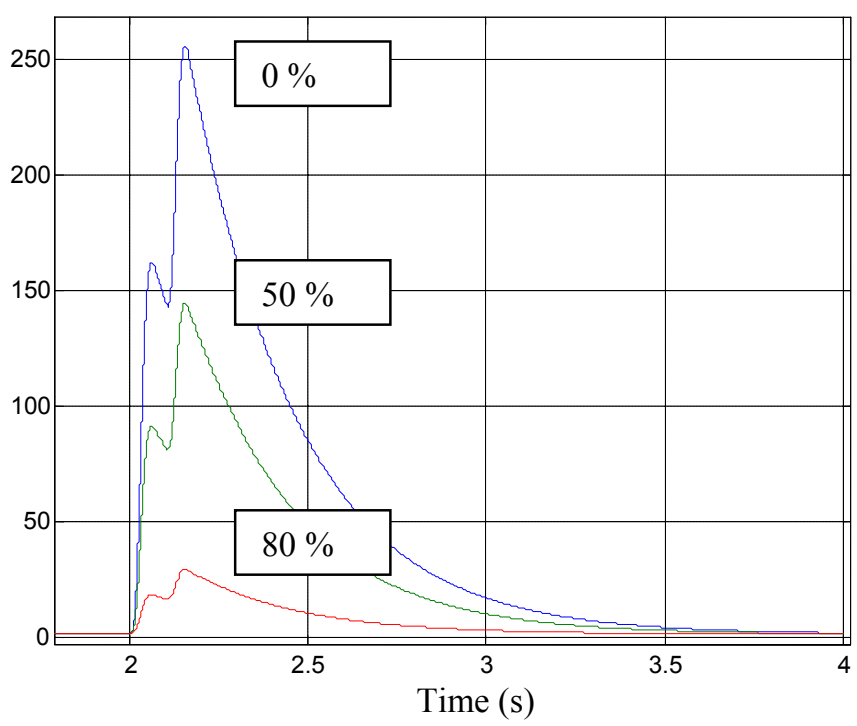

Fig. 4. IFL transient for a $0.1 \mathrm{~s}$ sags.

The methodology proposed to carry out the test, is as follows:

1. To define the waveform to be generated. This waveform is a $50 / 60 \mathrm{~Hz}$ sinewave of nominal rms value.

2. To register the flicker levels Pst and Plt in steady state.
3. To generate the programmed sags.

4. To register the flicker levels Pst and Plt for that short/long time period again.

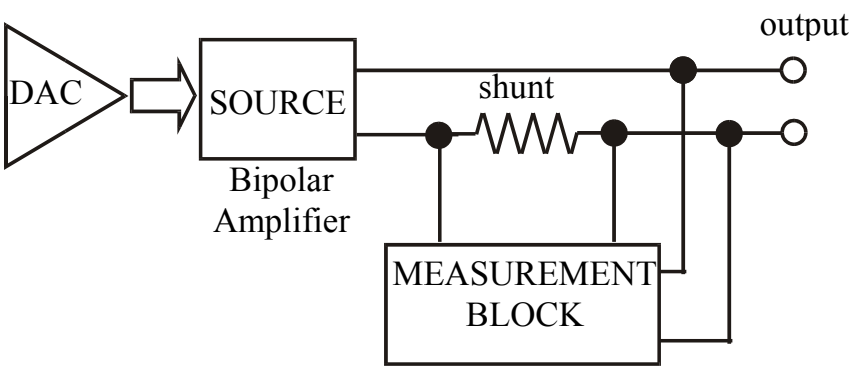

Powermeter

FFT Analyzer

Fig. 5. AC Source Functional Elements.

All the flickermeters under test are connected to the same point so they are measuring the same voltage.

\section{Results}

The proposed test system and methodology has been applied to the commercial flickermeters compiled in Table II. All the instruments are sensitive to sags. Figures 6, 7 and 8 show time evolution of Pst.

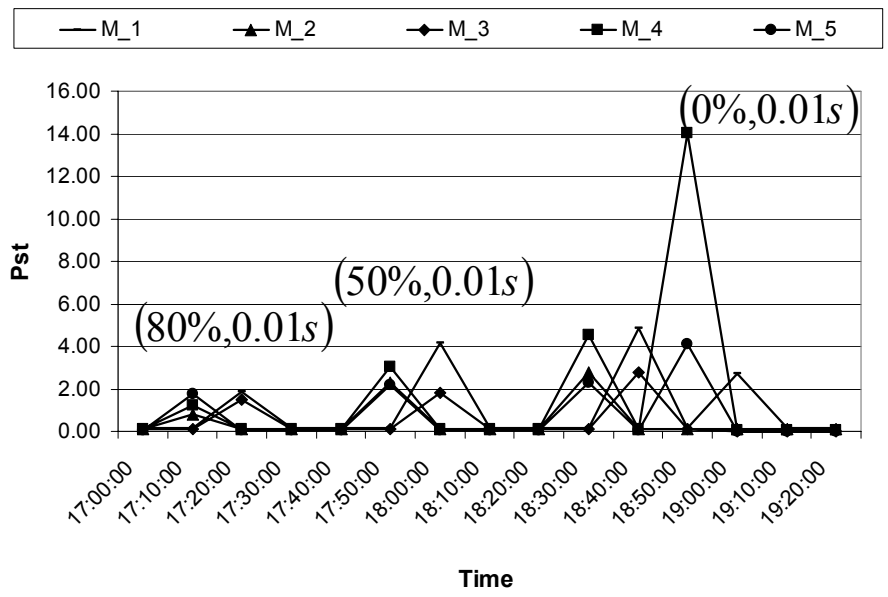

Fig. 6. $0.01 \mathrm{~s} \mathrm{sag}$ influence on Pst for various flickermeters.

\section{Conclusions}

The paper has shown the sensitivity that flickermeters designed according EN 61000-4-15 [5] have to voltage sags. A flickermeter model has been implemented in order to simulate the influence of voltage sags. From an experimental point of view, five different commercial flickermeters have been put to the test. The work has also shown how a single sag of short duration modifies the 10 minutes Pst. 


-M_1 $\rightarrow$ M_2 $\rightarrow$ M_3 $\rightarrow$ M 4 $\rightarrow$ M 5

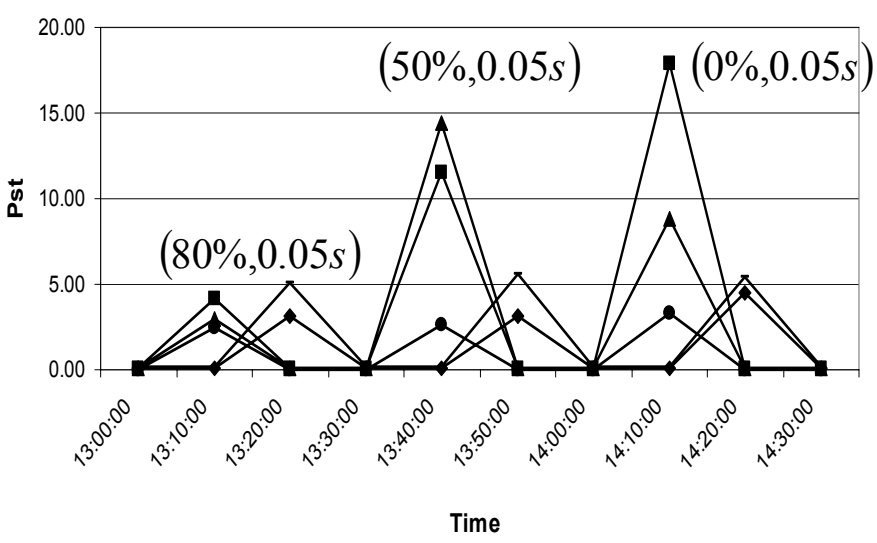

Fig. 7. $0.05 \mathrm{~s}$ sags influence on Pst for various flickermeters.

It is important to highlight that flicker measurement should also include sags and swells in order to discard Pst values computed during time intervals with sags and swells. In addition, flicker measurements without simultaneous sags and swells registration can be questioned.

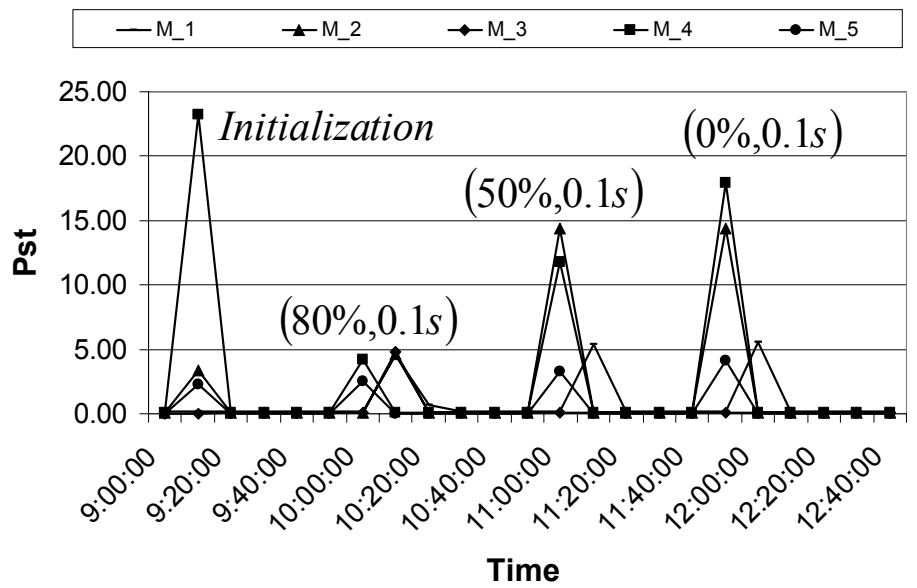

Fig. 8. 0.1 s sags influence on Pst for various flickermeters.

\section{Acknowledgement}

The authors wish to acknowledge the technical support of the VIESGO (ENEL) Power Quality staff.

\section{References}

[1] CENELEC EN 50160.Voltage characteristics of electricity supplied by public distribution systems. 1999.

[2] CENELEC EN 61000-4-7. Electromagnetic compatibility (EMC) -- Part 4-7: Testing and measurement techniques - General guide on harmonics and interharmonics measurements and instrumentation, for power supply systems and equipment connected thereto. 1997.

[3] CENELEC EN 61000-3-3. Electromagnetic compatibility (EMC) -- Part 3-3: Limits - Limitation of voltage changes, voltage fluctuations and flicker in public low-voltage supply systems, for equipment with rated current $<=16$ A per phase and not subject to conditional connection. 1995.

[4] Koponen, P.; Makinen, A.; Seesvuori, R.; Voltage dips cause problems with digital flickermeters. IEEE Power Tech Conference. Vol. 1. September 10-13, Porto, Portugal. 2001.

[5] CENELEC EN 61000-4-15. Electromagnetic compatibility (EMC) -- Part 4-15: Testing and measurement techniques - Flickermeter - Functional and design specifications. 1998.

[6] IEC 868. IEC/TR2 60868 (1986-09). Flickermeter Functional and design specifications.

[7] McKim, J.; The UIE Flickermeter Demystified. http://www.cemag.com/archive/1999/mayjune/McKim.html\#.

[8] Using Matlab 6.0 - Simulink 5.0. Mathworks. 2002.

[9] HP6834B. User's Guide. Agilent Technologies. 2000. 